\title{
Supervisão em Terapias Cognitivo-Comportamentais: Trilhando outros Caminhos Além do Serviço-Escola
}

\author{
Conceição Reis de Sousa - Universidade Federal de São Paulo, Santos, Brasil \\ Ricardo da Costa Padovani - Universidade Federal de São Paulo, Santos, Brasil
}

\begin{abstract}
Resumo
O objetivo deste estudo é descrever um modelo de supervisão que prepara psicólogos para desenvolver ações voltadas para prevenção, promoção, proteção e reabilitação em saúde. A orientação teórica deste artigo é da abordagem cognitivo-comportamental, dialogando com as noções de cuidado integral e de trabalho em equipe interprofissional. Os estudos sobre essa articulação são escassos, dificultando o compartilhamento dessas práticas de formação. A oportunidade de descrever esse modelo ampliado de formação de terapeutas cognitivo-comportamentais aponta uma das várias possibilidades de preparação do aluno do curso de Psicologia e indica a necessidade de pesquisas sistemáticas na área.

Palavras-chave: supervisão clínica, estudantes universitários, psicologia, terapia cognitivo-comportamental
\end{abstract}

\section{Supervision in Cognitive Behavioral Therapy: Tracing other Ways Besides the School Service}

\begin{abstract}
The objective of this study is to describe a supervision model that prepares psychologists to promote actions at prevention, promotion, protection and health rehabilitation. The theoretical orientation of this article is the cognitive-behavioral approach that considers the concepts of comprehensive care and work in inter-professional team. Studies about this topic are scarce, making it difficult to share these training practices. The opportunity to describe this model of cognitive-behavioral therapist's formation shows the various possibilities of preparation of student of psychology and indicates the need for systematic research in the area.
\end{abstract}

Keywords: clinical supervision, college students, psychology, cognitive behavioral therapy

\section{Supervisión en Terapias Cognitivo-Conductuales: Utilizando otras Vías Además del Servicio de la Escuela}

\begin{abstract}
Resumen
El objetivo de este estudio es describir un modelo de supervisión que prepara psicólogos para desarrollar acciones dirigidas a la prevención, promoción, protección y rehabilitación de la salud. La orientación teórica del artículo es el enfoque cognitivo-conductual que dialoga con las nociones de cuidado integral y el trabajo en equipo interprofesional. Los estudios sobre este tema son escasos, por lo cual es difícil compartir estas prácticas de formación. La oportunidad de describir este modelo ampliado de formación de terapeutas cognitivo-conductuales muestra las diversas posibilidades de preparación de los estudiantes de Psicología e indica la necesidad de investigaciones sistemáticas en el área.

Palabras clave: supervisión clínica, estudiantes universitarios (psicología), terapia cognitivo-conductual
\end{abstract}

A mudança na concepção de saúde, marcada na Constituição de 1988, exigiu reformulação das ações e da formação dos psicólogos. Sendo profissional da saúde, como definido pelo Conselho Nacional de Saúde (resolução CNS no 218/97), o psicólogo deve desenvolver competências para ações voltadas para prevenção, promoção, proteção e reabilitação em saúde (Carvalho, Freire \& Bosi, 2009). As Diretrizes Curriculares Nacionais do Curso de Psicologia destacam a necessidade de desenvolver recursos para trabalhar em equipes multiprofissionais, em diferentes contextos, na perspectiva do cuidado integral (Brasil, 2002). Dessa forma, a formação do psicólogo assume o compromisso com as mudanças no campo da saúde, o que passa pelo questionamento do modelo clínico tradicional. Para além dos dispositivos legais, a renovação do modelo clínico tradicional exige a experimentação de novos métodos de ensino-aprendizagem, bem como a superação do modelo clínico centrado no atendimento individual e na clínica privada (Luiz Ribeiro \& Amélia Luzio, 2008).

O objetivo deste artigo teórico é descrever um modelo de supervisão clínica na abordagem cognitivo-comportamental. A proposta, seguindo as recomendações do MEC, do CNS e do próprio Projeto Político Pedagógico (PPP) da universidade, é que o processo de ensino-aprendizagem da Terapia Cognitivo-Comportamental (TCC) venha acompanhado de recursos que fortaleçam as ações interprofissionais na perspectiva da integralidade do cuidado. A TCC, como as demais abordagens teóricas, pode ser um instrumento de transformações individuais e coletivas, para que tal potencial se concretize, é preciso atentar para as novas exigências na formação dos futuros terapeutas. 
Há vários modos de desenvolver as competências para ser terapeuta, mas como apontado por Barletta, Fonseca e Delabrida (2012), os estudos sobre o tema são escassos, dificultando o compartilhamento dessas práticas de formação. Supervisores, independentemente da orientação teórica, precisam estar atentos às transformações do conceito de Psicologia Clínica e dos campos de estágio. A inserção em novos contextos, como o Sistema Único de Saúde (SUS), exigiu a adequação do desenvolvimento de competências e habilidades específicas para acolher o sofrimento humano, onde quer que se apresente, inclusive fora dos consultórios (Dutra, 2004).

O desenvolvimento das habilidades clínicas é uma exigência constante, pois, de acordo com os dados do Conselho Federal de Psicologia disponibilizado pelo Conselho Regional de Psicologia - SP (2013), em 2009, cerca de 80\% dos(as) psicólogos(as) brasileiros(as) exerciam a prática da Psicologia Clínica. O Conselho Federal de Psicologia (CFP) - autarquia responsável por regulamentar, orientar e fiscalizar o exercício profissional - definiu por meio da resolução do CFP $\mathrm{n}^{\circ}$ 013/2007 que o especialista em Psicologia Clínica é aquele que:

Atua na área especifica da saúde, em diferentes contextos, através de intervenções que visam reduzir o sofrimento do homem, levando em conta a complexidade do bumano e sua subjetividade. Estas intervenções tanto podem ocorrer a nivel individual, grupal, social ou institucional e implicam em uma variada gama de dispositivos clínicos já consagrados on a serem desenvolvidos, tanto em perspectiva preventiva, como de diagnóstico ou curativa (CFP, 2007, p. 24).

No entanto, é preciso admitir também que não há consenso entre os profissionais sobre o conceito de clínica, conforme apontado por Lohr e Silvares (2006), alguns argumentam que o método clínico relaciona-se com a capacidade de problematizar os fenômenos e levantar aspectos psicológicos envolvidos neles, independentemente do contexto; por outro lado, há os que consideram que método clínico está atrelado a modelo individualista.

A coexistência desses modelos de atuação clínica remete há um movimento de mudança na Psicologia, que ocorreu na década de 1980, quando a prática clínica deixa de ser focada apenas nos consultórios e se amplia para atenção básica e secundária. Nesse período, surgem novos referenciais teóricos que também se expandem para incluir a "inserção social do sujeito" (Brasileiro \& Souza, 2010). Alguns referenciais teóricos passam a explicar a subjetividade como produto de uma construção social e histórica, em oposição às práticas clínicas individualistas.

Contribuindo para a transformação do modelo clínico, as Diretrizes Curriculares Nacionais (2004) para a área de Psicologia rompem com a dicotomia teoria e prática. Nessa direção, Abdalla, Batista e Batista (2008) apontam que o deslocamento das diretrizes curriculares, baseadas em disciplinas para diretrizes alicerçadas em competências e habilidades profissionais, exigiu que docentes reestruturassem as atividades acadêmicas, inclusive as supervisões clínicas. O desenvolvimento das competências para o exercício da clínica, na perspectiva da terapia cognitiva, depende muito das supervisões clínicas (Barletta et al., 2012). Estudo realizado por Rangé, Falcone e Sardinha (2007) também aponta o treinamento em clínicas-escola, como instrumento fundamental para o desenvolvimento de competências básicas para o exercício da TCC.

A preparação para ser terapeuta cognitivo-comportamental pode ocorrer a partir de diferentes propostas didáticas, que podem ser mais ou menos alinhadas com o modelo tradicional de clínica. Quando as TCCs surgiram no Brasil, no final da década de 1980 predominantemente, no Rio de Janeiro e São Paulo, o modelo de atuação era centrado no indivíduo. $\mathrm{Na}$ década de 1990, em função de diversas evidências científicas de sua eficácia, o modelo propagou-se pelo país. Além da expansão regional, houve uma ampliação dos cenários profissionais na rede pública de saúde. Os novos caminhos trilhados solicitaram desenvolvimento de novas habilidades terapêuticas em TCC. Nas universidades, os futuros psicoterapeutas recebem formação generalista, de caráter apenas introdutório à psicoterapia, ainda que, a rigor, o estagiário, ao concluir o curso, possa trabalhar com psicoterapia sem ser especialista (Quayle, 2010). Logo, na graduação, não há proposta de formar especialistas em TCC, contudo é esperado que as competências e habilidades básicas e específicas sejam desenvolvidas ao longo do curso.

\section{O Processo de Aprendizagem das Habilidades Especificas da TCC}

De acordo com o Conselho Federal de Psicologia (2013), o estágio representa a área compartilhada entre as atividades acadêmicas e o treinamento profissional. O serviço-escola se insere no Projeto Político Pedagógico (PPP) de cada curso de Psicologia. O PPP estabelece, como objetivo geral, a preparação de psicólogos capazes de atuar em equipe interprofissional 
na perspectiva do cuidado integral (Universidade Federal de São Paulo, 2013). É evidente a necessidade de treinar profissionais capazes de atuar criticamente em diferentes contextos articulados com a realidade social (CFP, 2002). Apesar dessa demanda, ainda é pequeno o número de cursos de Psicologia que tenham conteúdos que abordem diretamente temáticas relativas ao SUS e às políticas públicas de saúde (Souto, Batista, \& Alves Batista, 2014; Luiz Ribeiro \& Amélia Luzio, 2008). A mudança teórica deve ser acompanhada por uma atitude crítica e, para tal, é necessário pensar novas formas de oferecer supervisão.

A inserção do estágio fora dos serviços-escola representa a ruptura da formação tradicional. A descrição de um modelo clínico, na perspectiva da TCC, articulado com a proposta de cuidado integral e em equipe interprofisisonal, representa uma contribuição para a preparação de novos terapeutas que possuam habilidades para a atuação no SUS, sem desconsiderar as especificidades da TCC. A perspectiva da integralidade deve contribuir para a compreensão da complexa constituição da subjetividade, bem como para o planejamento de intervenções a fim de reduzir o sofrimento do sujeito.

Terapeutas cognitivo-comportamentais realizam intervenções para reduzir sofrimento a partir da compreensão da subjetividade humana, entendida como uma interação recíproca entre crenças, emoções, comportamentos, fisiologia e ambiente. O reconhecimento e questionamento de seu próprio conjunto de crenças têm papel central no manejo de seu sofrimento. Portanto, o aluno deve ser treinado para entender a relação entre as crenças, as emoções, os comportamentos, a fisiologia, o ambiente e os problemas apresentados pelo paciente, isto é, desenvolver a habilidade de fazer a conceitualização de pacientes que sejam atendidos nos serviços-escolas ou em qualquer outro campo de estágio.

A conceitualização pode ser considerada como o recurso mais importante para o planejamento e condução de uma intervenção adequada. Esta pode receber diversas designações, como conceituação, formulação ou enquadre cognitivo do caso, sempre remetendo a compreensão cognitiva do paciente (Neufeld \& Cavenage, 2010). O desenvolvimento da habilidade de conceituar diminui a chance de intervenções não críticas, como a aplicação de técnicas cognitivas e/ ou comportamentais de forma desconectada da compreensão de quais são as crenças disfuncionais do paciente e de como elas estão relacionadas com os problemas e as formas de lidar com eles (Neufeld \& Cavenage, 2010; Kuyken, Beck, \& Dudley, 2010). O desenvolvimento da capacidade de conceituar também ajuda que o terapeuta iniciante identifique a interferência de suas próprias crenças, gerando a urgência em mostrar sua competência por meio do uso de uma ou outra técnica como um indicativo do "saber como fazer". Ao longo da supervisão, é preciso deixar claro que a adequação de uma técnica está completamente relacionada a um apropriado entendimento da relação entre as crenças, as emoções, os comportamentos, a fisiologia, o ambiente (sua realidade social) e os problemas apresentados pelo paciente. O uso de técnicas deve ser precedido da capacidade de descrever e entender as dificuldades do paciente sem cindir os aspectos psicológicos do contexto social no qual o ele se encontra. Se não houver essa compreensão mais ampla, corre-se o risco de um tecnicismo descompromissado com a integralidade do cuidado.

O cuidado integral deve estar embasado em uma compreensão ampla do ser humano, ou seja, em uma conceituação, que permita reconhecer o papel ativo do sujeito na redução de seu sofrimento. Kuyken, Beck e Dudley. (2010) propuseram um novo modelo de conceituação cognitiva, baseado no modelo tradicional desenvolvido por Beck, no qual ocorre a síntese da experiência individual do paciente com a teoria e as pesquisas da TCC. O diferencial, nesse modelo proposto, é a valorização de três princípios norteadores dessa prática: o empirismo colaborativo, a incorporação de pontos fortes do cliente e o desenvolvimento de níveis de conceituação. A valorização da participação do paciente no entendimento de seus problemas, bem como de seus pontos fortes vai ao encontro dessa na forma de compreender a saúde proposta no PPP da universidade, pelo MEC e pelo CNS.

Baseado nesse modelo proposto por Kuyken et al. (2010), são descritas as práticas de supervisão que recorrem ao uso do empirismo colaborativo, da incorporação de pontos fortes do cliente e o desenvolvimento de níveis de conceituação para nortear o processo de formação de terapeutas.

\section{Empirismo Colaborativo}

A fim de formular uma compreensão sobre os problemas do paciente, pode-se recorrer ao empirismo colaborativo ou ao modelo explanatório unilateral, próprio dos modelos clínicos tradicionais. Trabalhar colaborativamente com o paciente favorece a adesão ao tratamento, bem como fomenta a autonomia. 
Terapeutas dispõem de teorias e pesquisas em TCC para descrever e explicar os problemas do cliente, mas é aquele que sofre que oferece dados fundamentais e feedback que ratificam ou não a conceitualização. A formulação compartilhada do caso é fundamental na escolha das metas terapêuticas e das intervenções a serem realizadas (Beck, 1997; Knapp \& Beck, 2008; Neufeld \& Cavenage, 2010).

Conceitualizar sem a real participação do paciente é comum a quem está iniciando a prática clínica, pois, apesar da existência de uma nova concepção de saúde centrada no sujeito, ainda é forte a tendência a focar na doença. (Guareschi, Reis, Ecker, \& Machry, 2014). Outro erro é não valorizar a forma como o paciente explica seus problemas. A discordância - explicita ou velada - em relação à descrição e explicação das queixas compromete a adesão ao tratamento. Portanto, não basta comunicar ao paciente a formulação do caso, é preciso discuti-la e mesmo retificá-la até que ela faça sentido para ambos. Outro desafio para alguns iniciantes é a integração entre as explicações para cada uma das queixas apresentadas, sua ausência também aponta dificuldades na formulação de caso e torna menos provável a aceitação da formulação pelo paciente (Kuyken et al., 2010).

Mesmo durante a supervisão, é preciso estar atento à arte de ensinar a conceitualizar de forma colaborativa. As limitações de tempo, o frágil domínio do modelo cognitivo pelos estudantes e a tendência à escuta desenvolvida ao longo da vida escolar dificultam o trabalho conjunto entre supervisores e estagiários. Sabe-se que a aprendizagem por modelação é muito importante. É possível fazer a analogia da relação supervisor/estagiário, e deste com seu paciente. $\mathrm{O}$ supervisor tem maior domínio das teorias, pesquisas e da clínica em TCC, mas é o aluno que presta o atendimento e que tem mais condições de oferecer dados para o entendimento do caso. Quando o estagiário participa mais colaborativamente da conceitualização, ele tende a refletir de forma mais crítica sobre a descrição e explicação dos problemas do paciente, ficando mais propenso a identificar e corrigir possíveis falhas no processo - o que contribui para o desenvolvimento do processo de aprendizagem reflexiva (Kuyken et al., 2010; Padesky 2004).

\section{A Incorporação de Pontos Fortes}

Outro ponto crítico é ensinar sobre a incorporação dos pontos fortes, ou seja, a identificação dos recursos atuais do cliente que podem ser usados para enfrentar a adversidade (Neufeld \& Cavenage, 2010).
Terapeutas iniciantes podem se concentrar apenas nas queixas, esquecendo-se dos recursos internos e externos. Esse princípio torna-se mais importante quando se atua em instituição da rede pública de saúde. É possível tomar como ilustração o caso dos adolescentes atendidos pelos estagiários em equipamento público de saúde. Muitos adolescentes oriundos de famílias desestruturadas enfrentam situações cotidianas de violência e frequentam uma escola que produz mais exclusão que inclusão. Apesar de tais adversidades, apenas uma parcela apresenta transtornos mentais. Pesquisa desenvolvida por Rozemberg, Avanci, Schenkere e Pires (2014) também aponta a ausência de nexo entre a forma de enfrentamento e condição socioeconômica e outras variáveis, como sexo e cor/raça. O modo de enfrentamento dos jovens não é uma condição inata, mas sim de uma característica que se desenvolve a partir da interação da pessoa com seu meio. Portanto, a forma de lidar com a adversidade não é uma condição estática, podendo ser fortalecida por profissionais da saúde e da educação, pelas famílias ou por qualquer grupo social ou instituição (Assis, Pesce, Avanci, \& Njaine, 2005). Nesse cenário, o fortalecimento desses recursos de enfrentamento remete ao compromisso dos terapeutas cognitivos com as necessidades dessa população mais vulnerável.

\section{Niveis de Conceitualização}

Segundo Kuyken et al.(2010), a conceitualização evolui, ao longo da terapia, do nível descritivo para o explanatório. Inicialmente, é necessário ajudar o estagiário a traduzir as queixas do paciente em termos de pensamentos, sentimentos e comportamentos. Essa tarefa exige que o estudante use a lente fornecida pelo modelo cognitivo para entender os problemas do paciente (conceitualização descritiva). Em seguida, é preciso identificar fatores desencadeantes e mantenedores do problema dos problemas atuais (conceitualização transversal) e, por fim, o reconhecimento de fatores predisponentes e dos protetores (conceitualização longitudinal). Progressivamente, a conceitualização torna-se mais complexa à medida que mais elementos são integrados (Neufeld \& Cavenage, 2010).

O desenvolvimento da capacidade de realizar os três níveis de conceituação exige um aprendizado sistemático da TCC. Há vários modelos explicativos que permitem discutir o desenvolvimento de competências e habilidades especificas da TCC. No formato proposto por Kuyken et al. (2010) há uma adaptação do modelo proposto por Bennett-Levy (2006) que descreve três 
processos de aprendizagem interligados: declarativo (quando o estudante adquire conhecimentos fundamentais, como as teorias da TCC ou protocolos de tratamento, isto é, o "saber que"), procedimental (habilidades para saber como conduzir a TCC, incluindo habilidades interpessoais e técnicas, ou seja, o "saber como") e reflexivo (envolvendo a capacidade de observar, analisar, avaliar e refletir sobre as próprias ações terapêuticas).

O processo declarativo é o meio pelo qual o aluno tem acesso à informação tanto sobre as teorias, técnicas e protocolos, bem como habilidades sociais relevantes para relação terapêutica. Esse processo de aprendizagem ocorre nas unidades curriculares de TCC, em participações em cursos e congressos e por meio de leituras especializadas. No entanto, a tradução desse conhecimento como competência ou habilidade (aspecto processual) não ocorre passivamente, são necessárias atividades práticas em contextos clínicos ou de supervisão (Bennett-Levy, 2006).

Kuyken et al. (2010) destacam ainda que a dimensão procedimental permite o desenvolvimento do "como" conduzir a TCC e abarca: habilidades técnicas (por exemplo, socializar o cliente ao modelo cognitivo ou realizar questionamento socrático); habilidades interpessoais (por exemplo, estabelecer o vínculo terapêutico) e habilidades não verbais (tal como, perceber alterações significativas no tom de voz).

O processo reflexivo envolve a capacidade de o terapeuta distanciar-se da própria prática e refletir criticamente sobre ela. São exigidas capacidades de observação, análise e avaliação dos conhecimentos declarativos e procedimentais empregados em cada caso. Bennett-Levy (2006) sugerem que, para desenvolver esse senso crítico, devem ser estimuladas estratégias, como terapia pessoal, o emprego do estilo socrático de supervisão e uso de aprendizagem baseada em problemas.

O avanço do processo declarativo para o procedimental e finalmente para o reflexivo é um dos grandes desafios na formação clínica, representando a integração entre o saber acadêmico e desenvolvimento das competências e habilidades profissionais.

\section{A Estrutura do Estágio}

Adotando como referência o modelo de ensino e aprendizagem proposto por Kuyken et al. (2010) para o desenvolvimento das competências de terapeutas de TCC são apresentadas as estratégias que buscam preparar futuros terapeutas brasileiros a usar a TCC para promover ações voltadas para prevenção, promoção, proteção e reabilitação em saúde.

O estágio de TCC nessa universidade é anual e realizado em dois campos: (a) instituição pública voltada ao atendimento de adolescentes e (b) Serviço-Escola de Psicologia (SEP). Esse formato cria oportunidade diferenciada para os estudantes, pois eles podem exercitar suas habilidades em ambiente institucional e em consultório. A duplicidade de campos enriquece a formação que, além do desenvolvimento de habilidades específicas da TCC, possibilita a aprendizagem do trabalho em equipe interprofisional.

\section{Evoluindo do "Saber que" para o "Saber como"}

O processo ensino-aprendizagem evolui gradativamente, a partir de um saber teórico até um fazer crítico (Kuyken et al. 2010; Neufeld \& Cavenage, 2010; Padesky, 2004). No início do estágio, é necessário fortalecer o processo declarativo por meio de leituras técnicas complementares, além daquelas já realizadas no módulo de Terapia Cognitivo-Comportamental (TCC). Os estagiários recebem formação teórica em diversas abordagens, por vezes, apresentam dificuldade de reconhecer a lente própria da TCC que foca nos processos cognitivos apontando como as próprias crenças da pessoa contribuem para a causa e/ou manutenção de seus problemas psicológicos. Mesmo quando as condições de vida são objetivamente desfavoráveis, a TCC pode contribuir para uma avaliação mais acurada da situação e para o desenvolvimento da capacidade de resolução de problemas (Moorey, 2004). Essa ressalva é importante porque grande parte da população atendida no equipamento público vive em condições adversas e de vulnerabilidade social. A fim de tornar a atuação profissional mais eficiente nesses contextos, é preciso articular noções como: cuidado integral e trabalho interprofisisonal aos conceitos da clínica na perspectiva da TCC.

Buscando ampliar a vivência dos alunos no modelo proposto, foram feitas discussões de caso e role-play a fim de desenvolver o conhecimento procedimental. Pensamentos automáticos relacionados à "desaprovação de supervisores e colegas" podem ter afetado a atuação dos alunos. A dificuldade em aceitar os próprios erros ou ainda a pouca familiaridade com o processo de encenar práticas podem ter inibido a participação nas primeiras atividades do role-play. Essa atividade possibilitou que os alunos refletissem criticamente sobre seu desempenho (embora o processo de aprendizagem reflexiva ainda estivesse insipiente). 
Após as práticas em campo, os estagiários faziam o relato verbal sobre as vivências no equipamento de saúde da prefeitura. Discutia-se sobre possíveis formas de compreender os problemas, bem como as intervenções feitas por outros profissionais, a partir da TCC. Por fim, eram feitas proposições de novas formas de intervir na situação quando ainda fosse possível retorná-la na semana seguinte. $O$ processo procedimental foi sendo consolidado por meio da atuação em campo.

\section{O Estágio em Equipamento Público Voltado ao Atendimento do Adolescente}

Um dos campos de estágio é um equipamento de saúde mental que atende adolescentes por meio de demandas espontâneas e referenciadas. Os problemas apresentados pelos adolescentes incluem transtornos mentais, queixas escolares, conflitos familiares e uso de drogas. A equipe interdisciplinar atua com uma proposta de atendimento integral e humanizado ao adolescente.

As ações dos estagiários foram ao encontro dos compromissos de transformação social assumidos pela Psicologia nos últimos anos (CFP, 2002) e da proposta da TCC de promover autonomia dos clientes (Cottraux \& Matos, 2007). As intervenções tinham como objetivo maior fortalecer recursos subjetivos dos adolescentes e seus familiares para que eles pudessem enfrentar a situação de vulnerabilidade social na qual estavam inseridos. A construção pelos jovens de novos significados é um recurso que pode ajudá-los a se perceber como agentes de sua própria vida, capazes de superar as vulnerabilidades biológicas e/ou sociais.

As intervenções clínicas devem ter como objetivo fomentar o crescimento pessoal e social dos sujeitos, tendo em vista todas as estruturas sociais fundamentais para a abordagem do problema (Cottraux \& Matos, 2007). Sendo assim, é preciso reconhecer que a realidade socioeconômica e cultural da população atendida no referido equipamento público é preocupante. Terapeutas cognitivos sabem que sintomas cognitivos, emocionais, comportamentais e fisiológicos não ocorrem no vácuo, mas dependem do contexto interpessoal/ambiente (Friedberg \& McClure, 2001).

O encaminhamento de adolescentes com problemas escolares, por exemplo, insere-se em uma realidade social que precisa ser considerada para uma compreensão mais acurada. Adolescentes com problemas na escola são rotineiramente encaminhados pelas escolas a esse equipamento público. Os encaminhamentos vêm acompanhados por solicitações de atendimentos pontuais que desconsideram os processos de tensão e desvalorização que adolescentes sofrem nas escolas. As implicações das desilusões vivenciadas nas escolas, a partir das frustrações das aspirações de ascensão social dos adolescentes e de suas famílias, não são reconhecidas. A desconsideração desse contexto pode levar à culpabilização exclusiva do adolescente pelos problemas de adaptação ao ambiente escolar (Rocha, 2008). O reconhecimento da contribuição do ambiente escolar para os problemas de aprendizagem e de comportamento exige uma conceitualização mais ampla dos problemas, favorecendo o desenvolvimento de novos recursos e o fortalecimento dos pontos fortes do adolescente para enfrentar problemas.

As "queixas escolares" dos adolescentes geraram a oportunidade dos estagiários avançarem no processo de aprendizagem procedimental. O exercício dessas habilidades foi consolidado em uma intervenção grupal realizada com seis adolescentes que haviam sido encaminhados para o referido equipamento por "problemas escolares". Foram realizadas inicialmente entrevistas individuais com cada adolescente e com seu responsável. Nesse momento, houve a oportunidade de desenvolver conceitualização de cada caso. Algumas das dificuldades encontradas pelos estagiários foram: (a) empregar o modelo cognitivo como lente para entender os relatos, (b) fazer questionamentos relevantes para a compreensão do funcionamento cognitivo, (c) desenvolver um vínculo que favorecesse a autoexposição do jovem e (d) estabelecer relação entre os diferentes problemas apresentados.

O passo seguinte foi o planejamento da intervenção com o grupo de adolescentes. A meta terapêutica foi auxiliar os participantes a "pensar sobre seu pensamento", isto é, identificar formas distorcidas de pensar e ampliar possibilidade de atribuir significado aos acontecimentos. A identificação da própria forma de pensar permite entender o porquê dos problemas atuais e modificar formas de enfrentamento (Caminha \& Caminha, 2008; Stallard, 2004). Foi feito um planejamento semanal, embora um dos estagiários não compreendesse a vantagem de um planejamento prévio por valorizar a espontaneidade das intervenções. Tal constatação foi ocasião para discutir a questão da estruturação das intervenções terapêuticas e das sessões. Foram levantadas as crenças sobre estruturação da ação do psicólogo, buscou-se elencar as vantagens e desvantagens, ampliando a concepção e o entendimento do planejamento prévio na TCC. Esse foi um 
ponto central no processo de supervisão, uma vez que foi trabalhado na perspectiva da construção da ativa e crítica do aluno.

A intervenção também incluiu orientação aos responsáveis, pois é fundamental estar atento ao vínculo estabelecido com o adolescente e com seus pais/responsáveis. Além disso, as relações familiares podem comprometer a saúde mental ou representar possibilidades de superação dos problemas, portanto, é preciso pensar no binômio adolescente/família. Rocha (2002) destaca que famílias, particularmente das classes menos favorecidas, dispõem de poucos recursos e informações, além da sobrecarga de trabalho e escassez de tempo para educar seus filhos, mas que tal situação pode ser manejada pelos profissionais de saúde.

O psicólogo, ao atuar com famílias, não pode perder de vista as condições de vida delas, sob o risco de apenas reproduzir a ação de culpá-las pelos problemas dos adolescentes. Terapeutas cognitivos sabem que não são os fatos ou pessoas que produzem sofrimento, mas o modo como elas interpretam as situações; essa significação é muito influenciada pelo meio social no qual cada um se insere (Beck, 1997; Knapp \& Beck, 2008). Reconhecendo esse contexto de vulnerabilidade social, foi oferecido orientação sobre práticas parentais positivas e os responsáveis foram incentivados a empregar as habilidades comportamentais e técnicas aprendidas no grupo para contribuir na superação dos problemas dos adolescentes.

As intervenções com os adolescentes e seus familiares foram realizadas em grupo em função das indicações das pesquisas sobre a eficácia das TCCs em grupo para o desenvolvimento de habilidades sociais e comportamentais (Caballo, 1999). As intervenções em grupo, com a participação de uma dupla de estagiários, facilitou o processo de aprendizagem do "saber fazer", pois propiciou a oportunidade de que os feedbacks ocorressem entre as próprias alunas.

Um obstáculo encontrado no avanço do conhecimento procedimental foi o desenvolvimento da habilidade de socializar os adolescentes e seus familiares ao modelo cognitivo. Constatou-se a tendência dos estagiários a explicar o modelo cognitivo de forma excessivamente didática e com pouco aproveitamento dos exemplos que surgiam no momento da socialização. Tal situação compromete o desenvolvimento da autonomia do usuário, que precisa aprender a compreender seus problemas a partir do modelo cognitivo. Outra habilidade específica desenvolvida no grupo foi o uso de técnicas cognitivas a fim de auxiliar os adolescentes a identificarem, questionarem e reestruturarem seu sistema de crenças.

As técnicas comportamentais também foram empregadas, pois a mudança do comportamento cria oportunidades de obter evidências factuais para testar a validade dos pensamentos, bem como podem gerar mudanças contingenciais importantes (Caminha \& Caminha, 2008). O manejo dessas técnicas foi difícil para os estagiários que, além do pouco domínio delas, precisavam adaptá-las à linguagem dos adolescentes. Finalmente o terceiro momento da formação, o processo de aprendizagem reflexiva, foi sendo desenvolvido à medida que algumas intervenções não tinham o efeito esperado ou que os estagiários percebiam suas próprias limitações do conhecimento "saber como".

A participação semanal, nas reuniões de equipe, propiciou a oportunidade de pensar sobre a importância do trabalho em equipe, pois nessas ocasiões foram discutidos os problemas dos adolescentes sob a perspectiva do cuidado integral e interprofissional. Um ponto importante na formação foi a orientação de que a avaliação ética incidia sobre as intervenções, e não sobre os profissionais que atuavam no equipamento.

\section{Serviço-Escola de Psicologia (SEP)}

Em ambiente mais tradicional, o SEP, o processo de evolução do "saber que" para o "saber como" possibilitou o desenvolvimento de habilidades específicas. O processo de aprendizagem procedimental em relação aos atendimentos individuais envolveu a compreensão de que realizar a conceituação cognitiva é diferente de diagnosticar. O diagnóstico remete a um conjunto de sintomas que descrevem de modo geral os problemas do paciente, sendo ateórico. A conceituação cognitiva é um conjunto de hipóteses personalizadas sobre os problemas da pessoa e é sustentada pelo modelo cognitivo (Friedberg \& McClure, 2007). É preciso compreender o que e por que é problema para o paciente, bem como reconhecer pontos fortes que poderão ser empregados para promover a autonomia. No SEP, a proposta de intervenção individual assumiu um caráter não apenas de tratamento dos sintomas da pessoa, mas de desenvolvimento da capacidade de gestão da própria vida, conforme proposto por Cottraux e Matos (2007).

A evolução do processo declarativo para o procedimental nem sempre foi fluida. $\mathrm{Na}$ supervisão, pode-se constatar a dificuldade dos estagiários em descrever as queixas do paciente em termos de relação 
entre pensamentos, emoções, comportamentos, fisiologia e ambiente. Essa dificuldade na conceitualização descritiva produzia efeitos indesejáveis no momento da socialização ao modelo cognitivo, dificultando a aprendizagem e adesão às técnicas propostas.

Outro obstáculo a ser contornado foi a dificuldade de distinguir entre questionar socraticamente e convencer o paciente de que ele está pensando de forma distorcida. A falta de experiência e a crença de que as mudanças terapêuticas devem acontecer prontamente podem ter contribuído para esse equívoco. Por outro lado, a realização de role-play, o levantamento de evidências confirmatórias ou contrárias aos pensamentos automáticos durante as supervisões, o reconhecimento da interferência das próprias crenças na forma de conduzir a técnica e outros recursos foram utilizados para minimizar essa falta de experiência do estagiário e ampliar o entendimento da aplicação do modelo cognitivo-comportamental.

Outro ponto central no processo de aprendizagem das habilidades terapêuticas foi a estruturação da sessão, aspecto discutido no manual técnico para clínicos em formação em terapia cognitiva proposto por Beck (1997). O estabelecimento de uma agenda exige domínio técnico e das habilidades interpessoais, incluindo a assertividade. A pouca familiaridade com a organização do tempo e das atividades pode ter contribuído para as dificuldades de estruturação da sessão. Foi sugerido aos estagiários questionar os próprios pensamentos automáticos ocorridos durante a sessão a fim de manejar essa dificuldade.

\section{Conclusões}

A apresentação desse modelo de supervisão de estágio clínico contribui para discussão sobre a formação de novos profissionais, incluindo as demandas oriundas da inserção de terapeutas que usam TCC no SUS. A passagem do processo "saber que" para o "saber como" precisa ser construída de forma colaborativa entre estagiários e supervisores. O desenvolvimento do processo reflexivo deve incluir a observação, análise e avaliação dos conhecimentos declarativos e procedimentais, bem como das implicações individuais e coletivas do uso desse saber para a promoção de saúde. Ao sair dos muros dos serviços-escola, a formação de novos profissionais da TCC toma contornos inovadores. Supervisores e estagiários assumem um compromisso com a transformação da rede de saúde por meio da oferta de intervenções qualificadas e contextualizadas.
A oportunidade de refletir e questionar o modelo de ensino-aprendizagem proposto neste artigo permite ampliar a discussão sobre essa importante etapa na formação do aluno do curso de Psicologia e aponta à necessidade de maior investimento em pesquisas sistemáticas na área. É preciso identificar quais propostas de formação podem ser mais benéficas para o desenvolvimento de terapeutas cognitivo-comportamentais comprometidos com cuidado integral e com o trabalho interprofissional.

\section{Referências}

Assis, S., Pesce, R. P., Avanci, J. Q., \& Njaine, K. (2005). Encarando os desafios da vida: Uma conversa com adolescentes. Rio de Janeiro: FIOCRUZ/ENSP/ CLAVES/CNPq.

Abdalla, I. G., Batista, S. H. S. S., \& Batista, N. A. (2008). Desafios do ensino de psicologia clínica em cursos de psicologia. Psicologia Ciência e Profissão, 28(4), 806-819. http://dx.doi.org/10.1590/ S1414-98932008000400012

Barletta, J. B, Fonseca, A. L. B., \& Delabrida, Z. N. C. (2012). A importância da supervisão de estágio clínico para o desenvolvimento de competências em terapia cognitivo-comportamental. Psicologia: Teoria e Prática, 14(3), 153-167. Recuperado de http:// pepsic.bvsalud.org/scielo.php?pid=S1516-36872012000300013\&script=sci_arttext

Beck, J. S. (1997). Terapia cognitiva: Teoria e prática (S. Costa, trad.). Porto Alegre: Artmed. (Original publicado em 1995).

Bennett-Levy, J. (2006). Therapist skills: A cognitive model of their acquisition and refinement. Behavioural and Cognitive Psychotherapy, 34, 57-8. doi: 10.1017/S1352465805002420

Brasil. (2002). Ministério da Educação. Diretrizes curriculares nacionais do curso de

graduação em psicologia. Brasil. Recuperado de http:// portal.mec.gov.br/cne/arquivos/pdf/CES0072. pdf

Brasileiro, T. S. A., \& Souza, M. P. R. (2010). Psicologia, diretrizes curriculares e processos educativos na Amazônia: Um estudo da formação de psicólogos. Revista Semestral da Associação Brasileira de Psicologia Escolar e Educacional, 14(1). doi: 10.1590/ S1413-85572010000100012 
Caballo, V. E. (1999). Manual de técnicas de terapia e modificação do comportamento. Santos: Santos Livraria Editora

Caminha, R. M., \& Caminha, M. G. (2008). Baralho das emoções: Acessando a criança no trabalho clínico. Porto Alegre: Sinopsys.

Carvalho, L. B., Freire, J. C., \& Bosi, M. L. M. (2009). Alteridade radical: Implicações para o cuidado em saúde. Physis: Revista de Saúde Coletiva, 19(3), 849865. doi: 10.1590/S0103-73312009000300017

Conselho Federal de Psicologia (2002). Adolescência e psicologia: Concepções, práticas e reflexões críticas. Brasilia: Conselho Federal de Psicologia.

Conselho Federal de Psicologia (2007). Resolução CFP N ${ }^{\circ}$ 013/2007. Brasília: Conselho Federal de Psicologia.

Conselho Federal de Psicologia (2013). Carta de serviços sobre estágios e serviços-escola. Brasília: Conselho Federal de Psicologia.

Conselho Regional de Psicologia SP (2013). Os caminhos da psicologia clínica. Psi, 176, 4-6.

Cottraux, J., \& Matos, M. G. (2007). Modelo europeu de formação e supervisão de terapia cognitivo-comportamentais para profissionais de saúde mental. Revista Brasileira de Terapias Cognitivas, 3(1), 54-72. Recuperado de http://pepsic. bvsalud.org/scielo.php?script $=$ sci_arttext\&pid $=$ S1808-56872007000100006

Dutra, E. (2004). Considerações sobre as significações da psicologia clínica na contemporaneidade. Estudos de Psicologia, 9(2), 381-387. doi: 10.1590/ S1413-294X2004000200021

Friedberg, R. D., \& McClure, J. M. (2001). A prática clinica de terapia cognitiva com crianças e adolescentes. Porto Alegre: Artmed.

Guareschi, N. M. F., Reis, C., Ecker, D. D., \& Machry, D. S. (2014). Formação em psicologia: O princípio da integralidade e a teoria da autopoiese. Revista Psicologia e Saúde, 6(1), 18-27. Recuperado de http:// www.gpec.ucdb.br/pssa/index.php/pssa/article/ viewFile/320/363

Knapp, P., \& Beck, A.T. (2008) Fundamentos, modelos conceituais, aplicações e pesquisa da terapia cognitiva. Revista Brasileira de Psiquiatria, 30(supl.II). doi: 10.1590/S1516-44462008000600002
Kuyken, W., Padesky, C. A., \& Dudley, R. (2010). Conceituação de casos colaborativa: $O$ trabalho em equipe com pacientes em terapia cognitivo-comportamental (Malllmann da Rosa, trad.) Porto Alegre: Artmed. (Original publicado em 2009).

Lohr, S. S., \& Silvares, E. F. M. (2006) Clínica-escola: Integração da formação acadêmica com as necessidades da comunidade. In E. F. M. Silvares (Ed.), Atendimento Clínico em Clínica-Escola (pp. 11-22). Campinas: Alínea.

Luiz Ribeiro, S., \& Amélia Luzio, C. (2008). As diretrizes curriculares e a formação do psicólogo para a saúde mental. Psicologia em Revista, 14(2), 203-220. Recuperado de http://periodicos.pucminas.br/index.php/psicologiaemrevista/article/view/350

Moorey, S. (2004). Quando coisas ruins acontecem a pessoas racionais: terapia cognitiva em circunstâncias adversas de vida. In P. M. Salkovskis (Ed.), Fronteiras da Terapia Cognitiva (pp. 235-255). São Paulo: Casa do Psicólogo.

Neufeld, C. B., \& Cavenage, C. C. (2010). Conceitualização cognitiva de caso: Uma proposta de sistematização a partir da prática clínica e da formação de terapeutas cognitivo-comportamentais. Revista Brasileira de Terapias Cognitivas, 6 (2), 3-35. doi: 10.5935/1808-5687.20100014

Padesky, C. (2004). Desenvolvendo competências do terapeuta cognitivo: modelos de ensino e supervisão. In P. M. Salkovskis (Ed.), Fronteiras da Terapia Cognitiva (pp. 235-255). São Paulo: Casa do Psicólogo.

Quayle, J. (2010). Reflexões sobre a formação do psicólogo em psicoterapia: Estado da arte e desafios. Psicologia Ensino \& Formação, 1(1) 99-110. Recuperado de http://pepsic.bvsalud.org/pdf/pef/ v1n1/09.pdf

Rangé, B. P., Falcone, E. M. O., \& Sardinha, A. (2007). História e panorama atual das terapias cognitivas no Brasil. Revista Brasileira de Terapias Cognitivas, 3(2), 53-68. doi: 10.5935/1808-5687.20070014

Rocha, M. L. (2002) Contexto do adolescente. In M. L. Contini, S. H. Koller \& M. N. S. Barros, (Ed.). Adolescência \& Psicologia: Concepscões, práticas e reflexões (pp. 25-32). Brasília: Conselho Federal de Psicologia.

Rocha, M. L. (2008). Inclusão ou exclusão? Produção de subjetividade nas práticas de formação. 
Psicologia em Estudo, 13(3) 477-484. doi: 10.1590/ S1413-73722008000300008.

Rozemberg, L., Avanci, J., Schenker, M., \& Pires, T. (2014). Resiliência, gênero e família na adolescência. Ciência \& Saúde Coletiva, 19(3), 673-684. doi:10.1590/1413-81232014193.21902013.

Souto, T. S., Batista, S. H., \& Alves Batista, N. (2014). A educação interprofissional na formação em Psicologia: Olhares de estudantes. Psicologia: Ciência e Profissão, 34(1), 32-45. doi: 10.1590/S1414-98932014000100004
Stallard, P. (2004). Bons pensamentos - bons sentimentos: | Manual de terapia cognitivo-comportamental para crianças e adolescentes. Porto Alegre: Artmed.

Universidade Federal de São Paulo (2013). Projeto político pedagógico do curso de graduação em psicologia. Recuperado de wmw2.unifesp.br/prograd/ app/cursos/index.php/prograd/arq_projeto/783

Recebido: $17 / 03 / 2014$

Primeira reformulação: 20/11/2014

Aprovado: 22/12/2014

Sobre os autores:

Conceição Reis de Sousa é mestre em Psicologia (UFRJ), psicóloga clínica no serviço-escola de Psicologia (UNIFESP), psicoterapeuta em consultório particular, foi supervisora em Psicologia Clínica, na linha cognitivo-comportamental, no Centro de Psicologia Aplicada da Universidade Paulista e atuou como psicóloga em Centro de Atenção Psicossocial (CAPS) em Guarujá.

E-mail: ceicaorsousa@ig.com.br

Ricardo da Costa Padovani é psicólogo, doutor em Educação Especial pela Universidade Federal de São Carlos, especialista em Terapia Comportamental Cognitiva em Saúde Mental pelo Instituto de Psiquiatria HCFMUSP/ Programa de Ansiedade e professor adjunto do curso de Psicologia da Universidade Federal de São Paulo - Campus Baixada Santista.

E-mail: ricardopadovani@yahoo.com.br

Contato com os autores:

Conceição Reis de Sousa

E-mail: ceicaorsousa@ig.com.br

Universidade Federal de São Paulo - Campus Baixada Santista

Serviço-Escola de Psicologia

Rua: Ana Costa, 178, Vila Belmiro, Santos/SP

CEP: $11.060-000$ 